\title{
Conociendo las funciones ejecutivas de los niños
}

\section{Knowing the executive roles of children}

\author{
Juan Manuel Canchumanya Popi*I \\ *Departamento Académico de Educación, Facultad de Educación, Universidad Nacional del Centro del Perú (UNCP), Huancayo-Perú.
}

\begin{abstract}
Resumen
Este artículo propone desde el método de la investigación acción, definir, dar identidad epistémica y orientar los procedimientos metodológicos, para valorar las funciones ejecutivas de los estudiantes, con apreciación de sus padres y profesores. El estudio se hizo en el nivel primario de una institución educativa pública. Desde el diseño, identificación, análisis del problema, formulación del plan de acción, recolección de la información, categorización y la estructuración de las nuevas categorías dieron lugar a las categorías emergente, el cual plantea alternativas de solución a los problemas de conducta y aprendizaje de los estudiantes. Entrenar las funciones ejecutivas a profundidad nos permitirá evitar trastornos patológicos en las personas.
\end{abstract}

Palabras Claves: Funciones ejecutivas, aprendizaje activo, psicología educativa, proceso cognitivo, inteligencia, educación basada en competencias.

\begin{abstract}
This article arises from the action research method, to define, give epistemic identity and guide the methodological procedures to know the executive functions of students, with the evaluation of their parents and teachers. The study was done at the primary level of a public educational institution. From the design, identification, analysis of the problem, formulation of the action plan, collection of information, categorization and structuring of the new categories, they gave rise to the emerging proposal, which raises alternative solutions to behavior and learning problems of the students. Training executive functions in depth will allow us to avoid pathological disorders in people.
\end{abstract}

Keywords: Executive functions, active learning, educational psychology, cognitive process, intelligence, competencebased education.

1 Correspondiencia: Juan Manuel Canchumanya Popi, jucanchumanya@gmail.com / neuroescuelatarma@gmail.com 


\section{Conhecendo os papéis executivos das crianças}

\section{Resumo}

Este artigo surge do método da pesquisa-ação, para definir, dar identidade epistêmica e orientar os procedimentos metodológicos para conhecer as funções executivas dos alunos, com a avaliação de seus pais e professores. O estudo foi realizado no nível primário de uma instituição de ensino pública. A partir da concepção, identificação, análise do problema, formulação do plano de ação, coleta de informações, categorização e estruturação das novas categorias, deram origem à proposta emergente, que levanta soluções alternativas para problemas de comportamento e aprendizagem dos alunos. O treinamento em profundidade das funções executivas nos permitirá evitar distúrbios patológicos nas pessoas.

Palavras Claves: Funções executivas, aprendizado ativo, psicología educacional, processo cognitivo, inteligencia, educacao baseada em competencias.

\section{Riqsispa chay ejecutivas ruwasqata warmakunapata}

\section{Uchuycha}

Kayartículumnin chay método de la investigación acción nisqan, rimanapaq, kanapaq epistémica hina, yuyaychaypaqprocedimientupi metodológicos yachanapaq imaynaruwanapaqyachaskuna, taytankuna, yachachiqkunaruraichikniptin. Yachaytamruwasqa nivel primario chay institución educativa públicapi. Haqaymantadiseño, identificación, análisis sasamanta, formulación chay plan cciónnisqan. uqarispahatunwillanata, categorizacionta chay estructuración musuq categoriaskunata, chaykunanhatarichinmañaqata emergentita, chaymihunmusuq paskatasasakunatapuriymanta, yachaymantallapallanyachaqkunata. Ruwaspa funciones ejecutivas ucukamachaymikanqachu trastornos patológicos runakunapi, allinllaspallapallancapacidasninta.

Hatun apup simi: Ejecutivas ruwasqata, llamkay Yuyay, llamchiy inhibitorio, riqsiy flexibilidad chay uyariy. 


\section{Introducción}

Hoy en día la ciencia ha descubierto que los cerebros y las mentes no nacen, sino que se construyen, en el centro de esta arquitectura dinámica se encuentra un conjunto de habilidades denominadas funciones ejecutivas. Las funciones ejecutivas son elementos claves para nuestro desarrollo durante toda nuestra vida por ende de nuestro aprendizaje.

Las funciones ejecutivas son habilidades de pensamiento que se desarrollan en nuestro cerebro, en los lóbulos frontales. Habitualmente, en el adulto, su disfunción entorpece la planificación y ejecución de conductas complejas, sin que por ello se afecten otros procesos perceptivos, motores o nemónicos de carácter más básico. Por ello, se considera a las funciones ejecutivas como el director del cerebro, porque nos permite reaccionar con respuestas comportamentales apropiadas a la situación, respuestas cognitivas propias del contexto. En todo momento tenemos las funciones ejecutivas en funcionamiento en el trabajo y en toda actividad que desarrollamos. Son tres las principales: la memoria de trabajo, el control inhibitorio y la flexibilidad cognitiva.

Urge trabajar en ellas desde tempranas edades, para lograr formar personas más ricas mentalmente, económicamente, prósperas, que tengan relaciones estables, alcancen el éxito, sean felices, sean buenos ciudadanos y que sean buenas personas.

La metodología investigación acción, según Miguelez (2004), permite abordar el estudio de la problemática situacional en contacto directo con los sujetos investigados y participar activamente en el planteamiento de la solución del problema. Al diseñar el proyecto se consideró realizar por etapas, esta primera etapa corresponde a valorar las funciones ejecutivas de los estudiantes del nivel primaria por parte de sus padres y profesores. Se identificó el problema luego de un análisis de la situación educativa actual, los rendimientos académicos y el nivel de violencia, observando los comportamientos de los estudiantes como se ven afectados. Luego del análisis, se observa comportamientos no favorables en los estudiantes y se decide implementar un plan de acción para identificar con precisión las razones de estas dificultades. Se revisa la literatura científica sobre este tema, concluyéndose en utilizar el cuestionario Behavior Rating Inventory of Executive Function, second edition (BRIEF 2), como instrumento de valoración de las funciones ejecutivas por parte de padres y profesores. Este cuestionario de adaptación española, fue validado por expertos psicólogos de nuestro país, quienes luego de adaptaciones concluyen en viabilizar su aplicación. De igual forma se seleccionó seis estudiantes del nivel primario; uno por sección, un niño y una niña por ciclo; coordinado con el consentimiento respectivo de padres y profesores se procedió a la recolección de información. Para dar lugar a la categorización de la información recolectada por el cuestionario que fue calificada en forma virtual por la plataforma de TEA Ediciones. Se continuó con la estructuración de las categorías, determinándose que son las dimensiones apriorísticas las que deben fortalecerse en su entrenamiento. A partir de la estructuración, surge una categoría emergente que se debe desarrollar. Finalmente se esbozan algunas conclusiones y recomendaciones.

\section{Diseño general del proyecto}

Los hechos visibles en educación, son una desproporcionada diferencia entre los que tienen mejores condiciones y quienes no las tienen, de igual forma nuestra sociedad mayoritaria expresa descontentos por múltiples circunstancias; y tal vez razón no les falte. En estudios de investigación científicas mundiales sobre satisfacción laboral la mayoría de ellos concluyen con altos porcentajes de insatisfacción laboral, para subsanar este problema implementan diversos programas para apalear esta crisis, Novoa (2019) indica en el campo empresarial "existe una relación positiva entre clima y satisfacción laboral". En el campo educativo en nuestro país "el 61,4\% muestran una baja satisfacción laboral" (Calderón y Raymundo, 2019) e incluso el 65,7\% muestran un moderado síndrome de burnout, estudio realizado en docentes en instituciones estatales. Los resultados en las evaluaciones ECE (2019) (evaluación de censo escolar), en segundo grado de primaria en lectura, en el nivel satisfactorio se alcanzó un 37,8\% de logro; en matemática se alcanzó 17\%. En la evaluación muestral 2019 a cuarto grado, se obtuvo 
en comunicación en el nivel de logro satisfactorio 34,5\%, en matemática se alcanzó en el nivel logro satisfactorio 34\%. En secundaria logro satisfactorio en matemática 17,7\%; en lectura 14,5\%; en ciencia y tecnología 9,7\%.

La violencia es otro punto a tener en cuenta, el 68,9\% de niños sufren violencia física y psicológica; el 54,9\% sufren violencia psicológica en el hogar y el 52,7\% violencia física; en adolescentes el 78\% sufren violencia familiar física y psicológica (INEI, 2019).

Por tanto, es necesario y posible implementar una nueva educación, que vaya más allá de los cognitivo que atienda las necesidades sociales, emocionales, físicas de todos los niños y adolescentes; que permita formar personas con un pensamiento complejo y abstracto, cualidad única de la especie humana, que resuelva y supere los problemas de manera creativa e innovadora. Esta nueva mirada nos la suministra la neuroeducación, que tiene un enfoque integrador, transdisciplinar y tiene como soporte las funciones ejecutivas, teniendo como objetivo mejorar los procesos de enseñanza y aprendizaje a partir de los conocimientos científicos alrededor del funcionamiento del cerebro.

\section{Identificación del problema}

Ante los diferentes hechos evidenciados en la sociedad, los estudiantes nos son ajenos y muestran diversas actitudes influenciados por estos temas: agresividad, intolerancia, incumplimiento de actividades falto de expectativas, mínima resiliencia, carentes de planificación para solucionar problemas, aspectos que limitan sus potencialidades como ser humano. Por ello, desarrollar las funciones ejecutivas es de marcada importancia; este constructo multidimensional tiene dominios diferentes pero todos interrelacionados y el desarrollo de todas ellas permitirá mejores aprendizajes en los estudiantes, fundamentalmente formar personas con más posibilidades de ser activos, proactivos y altamente ejecutivos en su vida cotidiana.

\section{Análisis del problema}

Las funciones ejecutivas desde el punto de vista de la investigación acción, lo expresamos como las actividades neuronales propias del lóbulo frontal. Identifican, analizan el problema y formulan un plan de acción (objetivo, acciones, tiempo, estrategia, canales, responsabilidades, recursos, e indicadores), recolectando, categorizando la información, estructuran las categorías, ejecutan el plan de acción, evalúan la acción ejecutada y plantean la propuesta estética emergente. Nos sirven para nuestra vida diaria, para adaptarnos eficazmente al entorno y alcanzar nuestros objetivos y metas.

Las dimensiones de las funciones ejecutivas, planteadas por Diamond (2013) son: "memoria de trabajo, control inhibitorio y flexibilidad cognitiva" (pág. 99). La memoria de trabajo es un elemento clave de las funciones ejecutivas, por lo cual la información se mantiene activa para la solución de problemas complejos que requieren múltiples pasos.

El control inhibitorio o autorregulación son capacidades para iniciar conductas, inhibir el efecto de ciertos estímulos, seleccionar objetivos relevantes para la tarea, planificar, organizar los medios cuando es necesario, mantener el plan, evitar distracciones y resolver conflictos. La flexibilidad cognitiva, pues no todo es control, también se da la autorregulación de las respuestas emocionales y conductuales, porque cada acción se evalúa, se vuelve a organizar y busca alcanzar objetivos, de esta manera se adapta el comportamiento de manera flexible cuando se enfrentan a problemas y situaciones novedosas.

Las evidencias empíricas describen que la inhibición es una habilidad de desarrollo temprano, mientras que la memoria de trabajo y la flexibilidad cognitiva tiene un curso de desarrollo más dilatado. Pero todos ello hoy se conoce que se pueden entrenar, cuanto más antes mejor. 


\section{Plan de acción}

Se planteó realizar las siguientes acciones para abordar este tema, se inició planteando el objetivo ¿Cómo valorar las funciones ejecutivas de los estudiantes del nivel primario por parte de sus padres y profesores de la Institución educativa integrada Antenor Rizo Patrón Lequérica de Condorcocha, Tarma?, se implementaron las siguientes acciones, seleccionar un estudiante promedio de cada grado, seleccionar y validar la prueba por expertos, coordinar la resolución personal del instrumento con las mamás y en forma virtual con las profesoras de cada grado, interpretar los resultados y plantear la propuesta emergente. La ejecución de todo el proceso fue autofinanciada por el autor sin compromisos con ninguna persona o entidad pública ni privada.

\section{Recolección de información}

Se procedió a la recolección de la información de seis estudiantes del nivel primario, tres varones y tres mujeres (un varón y una mujer por ciclo), los seleccionados fueron estudiantes promedios del aula: nivel de logro, comportamiento, apoyo de padres, condiciones económicas enfatizando que ninguno tuviese certificado clínico conductual o físico.

Se utilizó la prueba BRIEF2 para padres y profesores, este instrumento valora las funciones ejecutivas, fue validada con psicólogos expertos, en: sus atributos, redacción, ítems y propiedades psicométricas; quienes sugirieron adaptaciones en redacción, se subsanó las indicaciones y concluyeron en su valoración como instrumento viable para su aplicación. Se desarrolló el cuestionario en forma presencial con seis madres de familia por espacio de quince minutos en promedio y en forma virtual a sus profesoras por espacio de trece minutos en promedio.

\section{Categorización}

Los índices de cada evaluado se obtuvieron del sistema informático de TEA Ediciones (Técnicos especialistas asociados de psicólogos - España), los valores obtenidos se comparan con un criterio externo de referencia que expresa una puntuación típica, que es obtenida de la evaluación de un amplio grupo de individuos con características similares (edad, sexo, etc.), constituyéndose en un baremo de comparación. Para esta prueba se denomina puntuaciones $\mathrm{T}$ y tienen las posibles interpretaciones:

$\mathrm{T} \geq 70$, elevación clínicamente significativa;

T entre 65 y 69, elevación potencialmente clínica;

T entre 60 y 64, elevación leve;

T entre 0 y 59, sin significación clínica aparente.

De la información recolectada en el cuestionario para profesores (escuela), se obtuvo:

Tabla 1. Información recolectada en el cuestionario para profesores (escuela).

\begin{tabular}{|c|c|c|c|c|c|c|c|c|c|c|}
\hline ESCALAS ESTUDIANTES & INHIBICIÓN & $\begin{array}{l}\text { SUPERVISIÓN } \\
\text { DE SÍ MISMO } \\
\end{array}$ & FLEXIBILIDAD & $\begin{array}{c}\text { CONTROL } \\
\text { EMOCIONAL } \\
\end{array}$ & INICIATIVA & $\begin{array}{c}\text { MEMORIA DE } \\
\text { TRABAJO }\end{array}$ & PLANIFICACIÓN & $\begin{array}{l}\text { SUPERVISIÓN } \\
\text { DE LA TAREA }\end{array}$ & $\begin{array}{c}\text { ORGANIZACIÓN DE } \\
\text { MATERIALES }\end{array}$ & ÍNDICE GLOBAL \\
\hline I mujer & 70 & 54 & 58 & 65 & 48 & 68 & 64 & 66 & \begin{tabular}{|l|}
67 \\
\end{tabular} & 67 \\
\hline 2 mujer & 53 & 59 & 53 & 64 & 40 & 55 & 56 & 47 & 47 & 54 \\
\hline 3 mujer & 60 & 71 & 68 & 69 & 79 & 64 & 69 & 63 & 75 & 72 \\
\hline I varón & 43 & 49 & 41 & 57 & 39 & 42 & 45 & 41 & 43 & 43 \\
\hline 2 varón & 54 & 59 & 63 & 43 & 59 & 56 & 43 & 44 & 42 & 58 \\
\hline 3 varón & 51 & 61 & 65 & 56 & 45 & 64 & 53 & 53 & 54 & 59 \\
\hline
\end{tabular}

Plataforma TEA ediciones (2020)

De la información recolectada en el cuestionario para padres (familia), se obtuvo: 
Tabla 2. Información recolectada en el cuestionario para profesores (familia)Información recolectada en el cuestionario para profesores (familia)

\begin{tabular}{|c|c|c|c|c|c|c|c|c|c|c|}
\hline ESCALAS ESTUDIANTES & INHIBICIÓN & $\begin{array}{l}\text { SUPERVISIÓN } \\
\text { DE SÍ MISMO } \\
\end{array}$ & FLEXIBILIDAD & $\begin{array}{c}\text { CONTROL } \\
\text { EMOCIONAL }\end{array}$ & INICIATIVA & $\begin{array}{c}\text { MEMORIA DE } \\
\text { TRABAJO }\end{array}$ & PLANIFICACIÓN & $\begin{array}{l}\text { SUPERVISIÓN } \\
\text { DE LA TAREA }\end{array}$ & $\begin{array}{c}\text { ORGANIZACIÓN DE } \\
\text { MATERIALES }\end{array}$ & ÍNDICE GLOBAL \\
\hline I mujer & 64 & 69 & 67 & 65 & 70 & 64 & 66 & 67 & 61 & 70 \\
\hline 2 mujer & 58 & 52 & 47 & 62 & 43 & 52 & 57 & 47 & 57 & 55 \\
\hline 3 mujer & 60 & 71 & 50 & 56 & 53 & 62 & 62 & 47 & 48 & 59 \\
\hline I varón & 46 & 52 & 49 & 51 & 38 & 55 & 44 & 56 & 47 & 48 \\
\hline 2 varón & 59 & 54 & 58 & 44 & 36 & 65 & 38 & 45 & 46 & 54 \\
\hline 3 varón & 56 & 48 & 63 & 72 & 68 & 65 & 64 & 47 & 52 & 62 \\
\hline
\end{tabular}

Plataforma TEA ediciones (2020)

\section{Estructuración de categorías}

Con la lectura de los índices globales, notamos que una niña muestra en apreciación de los padres y otra niña en apreciación de su profesora, niveles cualitativos con problemas o dificultades mayores que otras personas de su edad y sexo, considerándose clínicamente significativos. De igual forma se observan que las funciones ejecutivas con índices altos, son la memoria de trabajo, la inhibición cognitiva y la flexibilidad cognitiva.

El método utilizado es la investigación acción y las técnicas empleadas para recolectar la información fueron el análisis documental, el cuestionario BRIEF2, observación de los estudiantes por parte del investigador.

\section{Categorización}

La mayoría de niños muestran dificultad a realizar una secuencia o una rutina para cumplir sus actividades, no recuerdan un número de teléfono completo o de su casa, no terminan las tareas, se olvidan lo que están haciendo, se distraen constantemente, no tienen rutinas, son indicadores de falta de entrenamiento de la memoria de trabajo. La mayor parte de los niños actúan sin haber pensado antes, muestran impulsividad, dicen: "disculpa, no me di cuenta", actúan fuera de control, se quedan sin reacción ante algún suceso, no prestan atención a las indicaciones, son índices de ausencia de entrenamiento de la inhibición cognitiva. A muchos niños les cuesta aceptar maneras distintas de resolver problemas, no se acostumbran a situaciones nuevas, se resiste a cambiar de rutinas, se alteran por los cambios, quieren mantener los mismos juegos, siempre se distraen, son indicadores de carencia de entrenamiento de la flexibilidad cognitiva.

Podemos observar también que hay un indicador común entre ellos, "no terminan las tareas", "no prestan atención a las indicaciones" "siempre se distraen", "se olvidan lo que están haciendo", estos indicadores nos dan referencia que hay una dificultad de la atención. Entonces podemos considerar que la atención es parte de todas las funciones ejecutivas, como tal, la consideramos como una categoría emergente.

\section{Discusión de resultados}

En nuestro país aún no existen trabajos educativos para valorar las funciones ejecutivas con participación de los padres de familia y de los profesores, quedando como referente esta investigación para implementar estudios de las funciones ejecutivas en los estudiantes y en todas las personas. En investigaciones internacionales, referente al control inhibitorio concluyen que se mejora significativamente durante los años escolares iniciales, los niños de 4-6 años son más impulsivos y no se toman el tiempo necesario para evaluar su respuesta, lo que disminuye su precisión. Estas habilidades mejoran significativamente en niños de 6 a 10 años. Un estudio señaló que el avance más importante en la capacidad de detener una respuesta ocurre entre los 7 y 9 años (Brocki \& Bohlin, 2004). En la mayoría de las investigaciones se observa el efecto de la edad en tareas de inhibición hasta los 13 años, momento en el cual parecería alcanzar su techo (Davidson, 2006). 
La mayoría de los estudios señalan que entre los 6 y 8 años de edad se observan marcadas mejoras en flexibilidad cognitiva (Anderson, 2002; Diamond, 2002). Algunas investigaciones señalan que la capacidad de aprender de los errores, plantear estrategias alternativas y el cambio del foco de la atención continua durante la adolescencia (Anderson, 2002; Davidson, 2006; Flores-Lázaro, 2014).

Otro estudio señaló que la estructura modular básica de la memoria de trabajo está presente desde los 6 años de edad y que cada componente aumenta su capacidad hasta la adolescencia. Diversos estudios con escolares han observado mejoras significativas en el bucle fonológico de la memoria de trabajo entre los 7 y 13 años de edad (Diamond, 2002), observándose dos picos uno a los 8 y otro a los 12 años de edad (Brocki \& Bohlin, 2004). Respecto de la agenda viso espacial, se ha observado que si la tarea es mantener la información visual en la memoria de trabajo los niños alcanzan la maestría alrededor de los 9 años de edad.

\section{Conclusiones}

Finalmente se concluye que el presente estudio cumple con el objetivo de valorar las funciones ejecutivas de los estudiantes del nivel primario de la Institución Educativa Integrada "Antenor Rizo Patrón Lequérica" de Condorcocha, cumpliéndose con dar respuesta al problema de investigación. Se logró valorar las debilidades comportamentales y cognitivas de las funciones ejecutivas por parte de los estudiantes.

Al sistematizar las respuestas de las mamás y profesoras se logró identificar una categoría emergente transversal que es la atención de los estudiantes.

Implementar un programa de entrenamiento de las funciones ejecutivas en los estudiantes de educación básica.

De igual forma emprender talleres pedagógicos para la capacitación de profesores y padres de familia en el entrenamiento de las funciones ejecutivas. 


\section{Referencias Bibliográficas}

Calderón Arellano, W. Y., \& Raymundo Vivar, G. M. (2019, URI: http://repositorio.upla.edu.pe/handle/UPLA/1233). Satisfacción laboral y síndrome de Burnout en docentes de instituciones educativas estatales de Huancayo 2019.

Diamond, A. (2013). Funciones Ejecutivas. Recuperado de https://adelediamond.com/ 01/08/2020

G.A.Gioia, P.K.Isquith, S. C. Guy \& L. Kenworthy BRIEF 2. Evaluación Conductual de la función ejecutiva. Adaptación Española. TEA Ediciones 2017.

Instituto Nacional de Estadística e Informática, Programa ENARES (2019), Recopilado de: http://www.grade.org. pe/creer/archivos/ENARES-2019.pdf INEI - ENARES 2019, 01/08/2020.

Miguelez, M. M. (2004). Ciencia y arte de la metodología cualitativa.

Ministerio de Educación, Unidad de medición de la calidad (2019), Recopilado de: http://umc.minedu.gob.pe/ ece2019/, 01/08/2020.

Novoa Salazar, J. G. (2019). Relación del clima organizacional en una empresa de la construcción. 